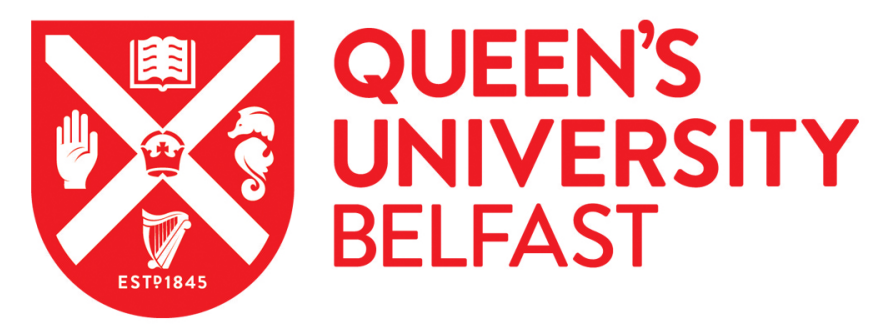

\title{
Measurement of Short-Range Correlations in Shock-Compressed Plastic by Short-Pulse X-Ray Scattering
} Barbrel, B., Koenig, M., Benuzzi-Mounaix, A., Brambrink, E., Brown, C. R. D., Gericke, D. O., Nagler, B., Rabec
Le Gloahec, M., Riley, D., Spindloe, C., Vinko, S. M., Vorberger, J., Wark, J., Wunsch, K., \& Gregori, G. (2009).
Measurement of Short-Range Correlations in Shock-Compressed Plastic by Short-Pulse X-Ray Scattering. Physical Review Letters, 102(16), [165004]. https://doi.org/10.1103/PhysRevLett.102.165004

Published in:

Physical Review Letters

Document Version:

Publisher's PDF, also known as Version of record

Queen's University Belfast - Research Portal:

Link to publication record in Queen's University Belfast Research Portal

Publisher rights

(C) 2009 The American Physical Society

\section{General rights}

Copyright for the publications made accessible via the Queen's University Belfast Research Portal is retained by the author(s) and / or other copyright owners and it is a condition of accessing these publications that users recognise and abide by the legal requirements associated with these rights.

Take down policy

The Research Portal is Queen's institutional repository that provides access to Queen's research output. Every effort has been made to ensure that content in the Research Portal does not infringe any person's rights, or applicable UK laws. If you discover content in the Research Portal that you believe breaches copyright or violates any law, please contact openaccess@qub.ac.uk. 


\title{
Measurement of Short-Range Correlations in Shock-Compressed Plastic by Short-Pulse X-Ray Scattering
}

\author{
B. Barbrel, ${ }^{1,2}$ M. Koenig, ${ }^{1}$ A. Benuzzi-Mounaix, ${ }^{1}$ E. Brambrink, ${ }^{1}$ C. R. D. Brown,${ }^{3,4}$ D. O. Gericke, ${ }^{5}$ B. Nagler, ${ }^{6}$ \\ M. Rabec le Gloahec, ${ }^{1}$ D. Riley, ${ }^{7}$ C. Spindloe, ${ }^{8}$ S. M. Vinko, ${ }^{6}$ J. Vorberger, ${ }^{5}$ J. Wark, ${ }^{6}$ K. Wünsch, ${ }^{5}$ and G. Gregori ${ }^{6,8}$ \\ ${ }^{1}$ Laboratoire pour l'Utilisation de Lasers Intenses, UMR7605, CNRS CEA, Université Paris VI Ecole Polytechnique, \\ 91128 Palaiseau Cedex, France \\ ${ }^{2}$ CEA, DAM, DIF, Ile-de-France, F-91297 Arpajon, France \\ ${ }^{3}$ Plasma Physics Department, AWE Aldermaston, Reading, United Kingdom \\ ${ }^{4}$ Plasma Physics Group, Blackett Laboratory, Imperial College, London, United Kingdom \\ ${ }^{5}$ Centre for Fusion, Space and Astrophysics, Department of Physics, University of Warwick, Coventry CV4 7AL, United Kingdom \\ ${ }^{6}$ Clarendon Laboratory, University of Oxford, OX1 3PU, United Kingdom \\ ${ }^{7}$ School of Mathematics and Physics, Queens University of Belfast, Belfast BT7 1NN, United Kingdom \\ ${ }^{8}$ Rutherford Appleton Laboratory, Chilton, Didcot OX11 OQX, United Kingdom
}

(Received 6 January 2009; published 23 April 2009)

\begin{abstract}
We have performed short-pulse $\mathrm{x}$-ray scattering measurements on laser-driven shock-compressed plastic samples in the warm dense matter regime, providing instantaneous snapshots of the system evolution. Time-resolved and angularly resolved scattered spectra sensitive to the correlation effects in the plasma show the appearance of short-range order within a few interionic separations. Comparison with radiation-hydrodynamic simulations indicates that the shocked plastic is compressed with a temperature of a few electron volts. These results are important for the understanding of the thermodynamic behavior of strongly correlated matter for conditions relevant to both laboratory astrophysics and inertial confinement fusion research.
\end{abstract}

DOI: 10.1103/PhysRevLett.102.165004

PACS numbers: 52.50.Lp, 52.25.Os, 52.27.Gr

The warm dense matter (WDM) regime, defined by close-to-solid mass densities $\left(\rho \sim \rho_{0} / 10\right.$ to a few $\left.\rho_{0}\right)$ and moderate temperatures $(T=1-10 \mathrm{eV})$, has been so far extremely challenging to model. Such a peculiar state of matter not only exhibits strongly nonideal plasma features, such as ion dynamics dominated by Coulomb interactions and liquid state short-range order, but also partly degenerate free electrons. The basic thermodynamics of WDM thus remains poorly described, despite its relevance in inertial confinement fusion [1], geophysics [2], and planetary astrophysics [3,4]. Since a few years ago, a new experimental technique based on $\mathrm{x}$-ray scattering [5] has allowed new insights into the microscopic structure of WDM states. In the case of spectrally resolved experiments [6-8], the scattered spectrum provides information on the electronic density $\left(n_{e}\right)$, temperature $(T)$, and degree of ionization $(Z)$, whereas angularly resolved experiments $[9,10]$ give insight into the ionic structure, the equation of state, and the spatial arrangements of ions and electrons. To date, the use of bright nanosecond long x-ray probe pulses has allowed the characterization of the hydrodynamic response of WDM states [10,11], on time scales which are essentially dictated by the spatial extent of the sample $(L \sim 100 \mu \mathrm{m})$ and the sound speed $c_{s} \sim$ $\left(k_{B} T_{i} / M\right)^{1 / 2}$ ( $M$ is the ion mass), thus giving $L / c_{s} \sim$ 1-10 ns. On the other hand, structural phase transitions (which involve global rearrangements of the ionic sites) occur on much faster time scales. Picosecond and subpico- second probes are thus required to observe snapshots in time of any significant change in the ionic correlations. Very recently, picosecond laser produced $K \alpha$ sources in spectrally resolved experiments have been used to investigate the insulator-to-metal phase transition in $\mathrm{LiH}$ [12]. In this work, we have instead implemented a novel ultrafast $K$ $\alpha$ source for angularly resolved scattering to measure the formation of short-range order at distances of a few Bohr radii as a result of strong coupling between the ions and the electrons.

Originally developed for liquid metals $[13,14]$ and later extended to the WDM regime [5,15], Chihara's approach to the modeling of $x$-ray scattering consists of writing the electronic structure factor as the sum of three terms $S_{e e}(k)=S_{\text {elas }}(k)+S_{\text {free }}(k)+S_{\text {trans }}(k)$, describing, respectively, the elastic scattering from (bound and free) electrons dynamically following to the ions, the highfrequency part of the free electron motion (giving rise to the plasmon feature in the collective scattering regime), and the resonant electrons undergoing a transition from a bound state under interaction with the incoming $\mathrm{x}$-ray photon. The resulting frequency-integrated scatter intensity is obtained as $I(k) \propto \sigma_{T} n_{i} S_{e e}(k)$, where $\sigma_{T}$ is the usual Thomson cross section, $n_{i}$ is the ion density, and the wave vector amplitude $k$ is related to the photon wavelength $\lambda_{0}$ and the scattering angle $\theta$ by $k=\frac{4 \pi}{\lambda_{0}} \sin (\theta / 2)$.

For the purpose of angularly resolved scattering from a dense low- $Z$ plasma, $S_{\text {free }}(k)$ and $S_{\text {trans }}(k)$ contribute only to 
a diffuse background, with the majority of the signal lying in the first term, which we can write as [14]

$$
S_{\text {elas }}(k)=\left|f_{I}(k)+\rho(k)\right|^{2} S_{i i}(k) .
$$

The terms $f_{I}(k)$ and $\rho(k)$ account for bound and free electron densities screening a nucleus, respectively; the ion-ion static structure factor $S_{i i}(k)$, related to the pair distribution function for the ions $g_{i i}(r)$, is the quantity that describes the extent of spatial correlations in the plasma. Different regimes are identified by the value of the ion coupling parameter $\Gamma_{\alpha \beta}=Z_{\alpha} Z_{\beta} e^{2} / 4 \pi \epsilon_{0} k_{B} a_{\alpha \beta} T$, where $\alpha, \beta=i, e$ (ions or electrons) and $a_{\alpha \beta}$ is the average interparticle separation. In the limit of small wave numbers $\left(k a_{i i} \ll 1\right)$, we can approximate $f_{I}(k) \sim Z_{A}-Z$ and $\rho(k) \sim Z$, where $Z_{A}$ is the atomic number of the ion nuclei. In the more general case of finite wave numbers, those two terms can be calculated as a function of the plasma temperature, density, and ionization state [5].

We now describe the experiment realized at the LULI 2000 facility. The frequency-doubled $(527 \mathrm{~nm})$, 1.5 ns-long laser beam was focused onto the front side of the $\mathrm{CH}$ (plastic) sample (see Fig. 1) in a 400- $\mu$ m-diameter flattop focal spot, using hybrid phase plates [16] and achieving a peak intensity of $I=5 \times 10^{13} \mathrm{~W} \mathrm{~cm}^{-2}$. To allow the compression of the plastic with minimal preheating from the radiating coronal plasma produced, the front face of the $\mathrm{CH}$ foil was coated with a multilayer substrate (that we refer to as the pusher) consisting of $\mathrm{CH}(15 \mu \mathrm{m}) / \mathrm{Al}(2 \mu \mathrm{m}) / \mathrm{Au}(0.5 \mu \mathrm{m}) / \operatorname{Al}(2 \mu \mathrm{m})$. The $\mathrm{x}$-ray probe radiation was generated by focusing the 30-ps-long, $1054 \mathrm{~nm}$ PICO 2000 chirped pulsed amplified beam line with an $f / 4$ off-axis parabola in a $100-\mu \mathrm{m}$-diameter focal spot onto a $10-\mu \mathrm{m}$-thick cop-

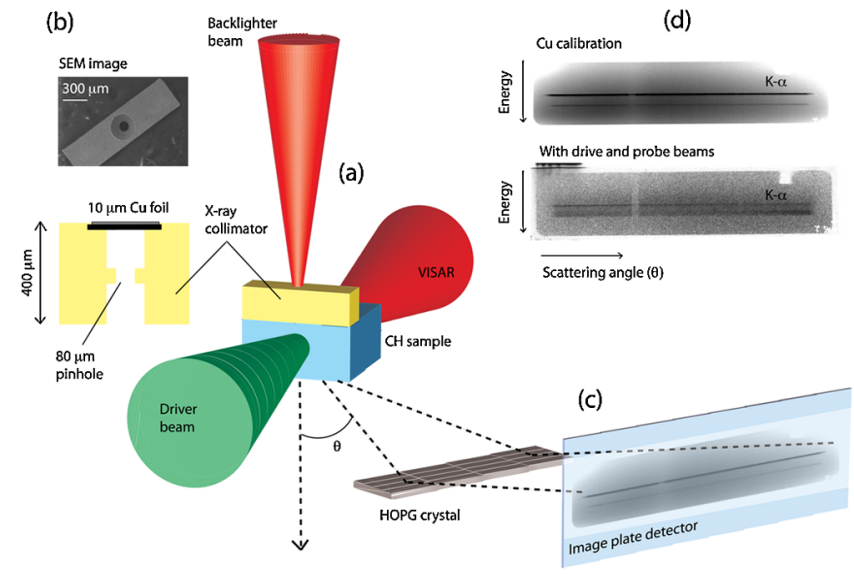

FIG. 1 (color online). Experimental setup. (a) Target assembly and beams layout; (b) $\mathrm{Au}$ x-ray collimator with $\mathrm{Cu}$ backlighter foil; (c) wide angle spectrometer with HOPG crystal and image plate; (d) example of experimental spectra. The $\mathrm{Cu}$ calibration shot was taken by replacing the target assembly with a single copper foil. It is used for spectral and angular calibration of the data. per foil. The interaction regime $\left(I \lambda^{2} \simeq 2.8 \times\right.$ $10^{16} \mathrm{~W} \mathrm{~cm}^{-2} \mu \mathrm{m}^{2}$ ) was chosen to optimize the generation of $\mathrm{Cu} K \alpha$ radiation $(E=8048 \mathrm{eV})$ [17]. The x-ray source was collimated into a $10^{\circ}$ half-aperture cone by a 80- $\mu$ m-diameter $\mathrm{Au}$ pinhole, transversely to the shock direction. The delay between the shock drive and the short-pulse backlighter was varied to access both the preshocked and the postshocked plastic. A 100-mm-wide highly oriented pyrolytic graphite (HOPG) ZYH-grade crystal $(2 d=6.7 \AA)$ collected the scattered x rays over a large angular range allowing in a single shot measurements to span from $\theta=25^{\circ}$ to $\theta=55^{\circ}$ scattering angles. Image plates were used as X-ray detectors. The mosaic spread of the HOPG crystal limits the intrinsic angular resolution to $3.5^{\circ} \pm 1.5^{\circ}$ [18], which is significantly smaller than the angular variation introduced by the Au pinhole.

Rear side time-resolved optical diagnostics (VISAR [19]) were used to calibrate the hydrodynamic simulations by measuring the shock breakout time at the pusher to sample interface. These were performed with the 1D radiative-hydrodynamics code MULTI [20], using SESAME tabulated equations of state [21]. Figure 2 shows the simulated time evolution of the plastic sample mass density from initial state $\left(\rho_{0}=1.03 \mathrm{~g} \mathrm{~cm}^{-3}, T_{0}=300 \mathrm{~K}\right)$. Our data were taken at two different probe-drive delays, which are also shown in the figure. It is clear that the temporal resolution provided by the 30-ps X-ray pulse allows selection of any probed plasma condition along the Hugoniot path followed by the target. On the other hand, the spatial resolution of the measurement is limited by modest collimation of the $\mathrm{x}$ rays, which was necessary to have a sufficient number of scattered photons. Mean density and temperature conditions for a given drive beam intensity and a probe-drive delay were extracted from the hydro-

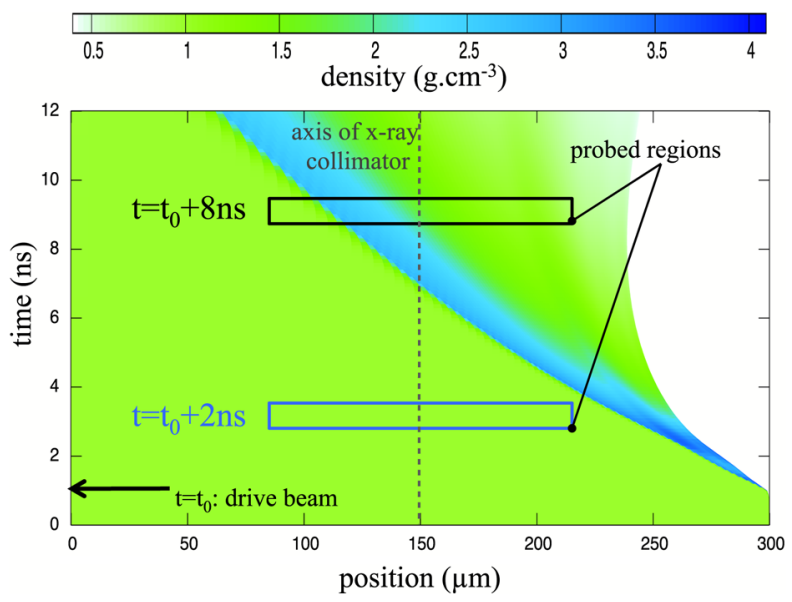

FIG. 2 (color online). Radiative-hydrodynamics simulation. The rectangular areas represent the probed region of the sample for the two experimental shots discussed in the text. The height of the rectangles (temporal resolution) is exaggerated for clarity. The laser drive illuminates the target from the right side of the figure starting at $t=t_{0}$. Only plastic cells are shown in the plot. 
dynamic simulations by averaging over the conical subvolume of the target traversed by the $\mathrm{x}$ rays. Corresponding values for the two delays shown in Fig. 2 are $\rho=$ $1.10 \mathrm{~g} \mathrm{~cm}^{-3}$ and $T=0.39 \mathrm{eV}$ for $t=t_{0}+2 \mathrm{~ns}$ and $\rho=$ $2.10 \mathrm{~g} \mathrm{~cm}^{-3}$ and $T=1.86 \mathrm{eV}$ for $t=t_{0}+8 \mathrm{~ns}$, where $t_{0}$ corresponds to the time the drive beam is fired onto the $\mathrm{CH}$ sample. Note that temperature averages are densityweighted to account for the fact that the scattering cross section is proportional to the number of scatterers. Besides, the width of the probed volume depicted in Fig. 2 is that of the cone at the midsection of the 500-m-wide target, so that even for early time some shocked plastic is in the field of view and contributes to the given temperature and density values.

The experimental elastic scattering data for the two probe-drive delays are shown in Fig. 3. We have compared the experimental points with the theoretical scattering profile generated by the model described in Ref. [22] which includes both strong-coupling effects and degenerate electron screening as well as higher order corrections to the dielectric response within a linear screening approximation [23]. While the elastic feature contribution is dominant, $S_{\text {free }}(k)$ and $S_{\text {trans }}(k)$ are accounted for as described in
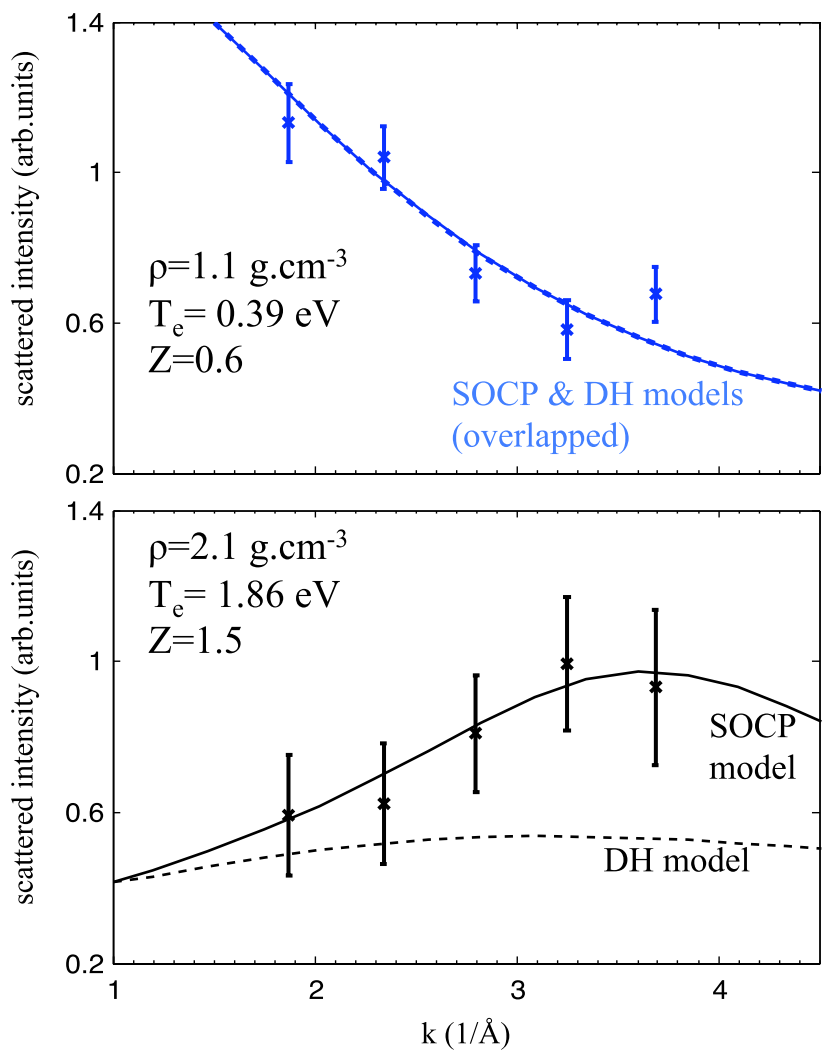

FIG. 3 (color online). Experimental data points and simulated spectra for $t=t_{0}+2 \mathrm{~ns}$ (upper panel) and $t=t_{0}+8 \mathrm{~ns}$ (lower panel). The coupling parameter is $\Gamma_{i i}=7.9$ for $t=t_{0}+2 \mathrm{~ns}$ and $\Gamma_{i i}=14.7$ for $t=t_{0}+8 \mathrm{~ns}$. SOCP and Debye-Hückel (DH) models are described in the text. Error bars represent the root mean square noise level in the recorded data.
Ref. [5]. The model only uses the temperature, density, and ionization state as input parameters, and it is referred to as the screened one-component plasma (SOCP) model. Given $T$ and $\rho$ from the MULTI simulation, the only remaining free parameter of the model is the ionization state $Z$, which was used as a fitting parameter. Best fit values for $Z$ and the corresponding simulated profiles for the two shots under consideration are shown in Fig. 3. In order to outline strong-coupling effects, the simple Debye-Hückel model [24] is also plotted in Fig. 3.

Since both $f_{I}(k)$ and $\rho(k)$ are monotonically decreasing with increasing wave number, the difference in the experimental data at $t=t_{0}+2 \mathrm{~ns}$ and $t=t_{0}+8 \mathrm{~ns}$ can be attributed to the formation of a correlation hole around each ion, characterized by small values of the ion-ion structure factor for $k \rightarrow 0$, as shown in Fig. 4. We have $S_{i i}(0) \sim\left(\kappa_{0} / \kappa+Z\right)^{-1}[22]$, where $\kappa_{0} / \kappa$ is the compressibility ratio ( $\kappa_{0}$ being the ideal isothermal compressibility) which is linearly increasing with $\Gamma_{i i}$ for large coupling parameters $\left(\Gamma_{i i} \gtrsim 50\right)$. We can estimate that for our conditions $S_{i i}^{t_{0}+2 \mathrm{~ns}}(0) / S_{i i}^{t_{0}+8 \mathrm{~ns}}(0) \approx 3.5$, and thus for the $t=$ $t_{0}+8 \mathrm{~ns}$ case the ion-ion structure factor is strongly suppressed at small wave numbers. Short-range correlations then appear as a broad peak centered at $k_{\text {peak }}=$ $3.2 \times 10^{10} \mathrm{~m}^{-1}$, corresponding to a correlation length $L_{\text {corr }}=1 / k_{\text {peak }} \sim 3 a_{i i}$. Indeed, this Letter provides the first compelling experimental evidence of incipient short-range electron order in high density matter.

MULTI predictions of the ionization state $\left(Z^{*}=0.04\right.$ and $Z^{*}=0.1$ for $t=t_{0}+2 \mathrm{~ns}$ and $t=t_{0}+8 \mathrm{~ns}$, respectively) are significantly different from the experimental fits. While the ionization table in MULTI is extracted from a hydrogenic calculation [25], for the same conditions of density and temperature, the average ionization deduced from the Thomas-Fermi model [26] is $Z_{\mathrm{TF}}^{*}=1.12$ and

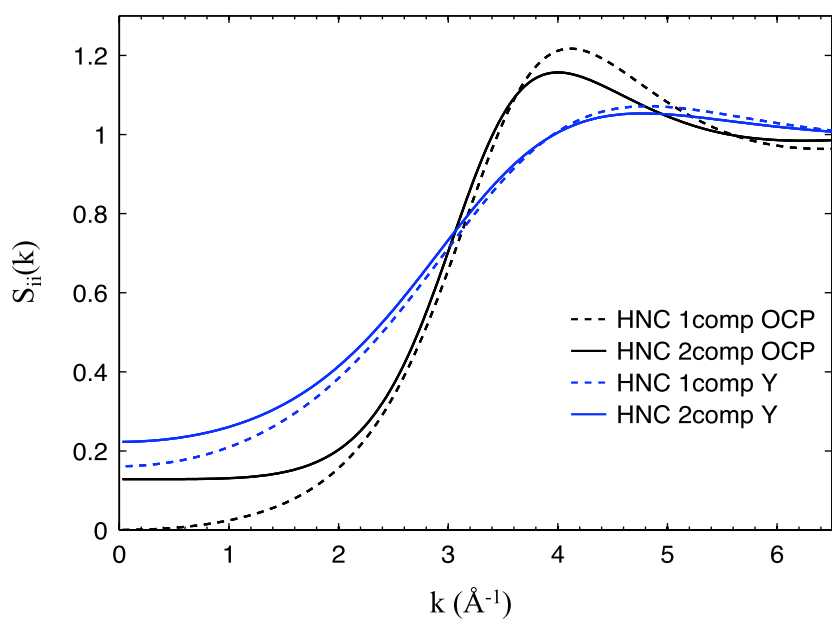

FIG. 4 (color online). Comparison between single-component and multicomponent HNC calculations for $S_{i i}(k)$. The plasma conditions refer to the case $t=t_{0}+8 \mathrm{~ns}$. The OCP calculations neglect screening, while $Y$-labeled lines use linear screening. 
$Z_{\mathrm{TF}}^{*}=1.36$ for $t=t_{0}+2 \mathrm{~ns}$ and $t=t_{0}+8 \mathrm{~ns}$, respectively. Since the ionization degree $Z$ as extracted from $x$-ray scattering refers to kinematically free electrons and valence electrons as well as delocalized electrons that are allowed to hop from one ionic site to another [27], we assume, as discussed by Dharma-Wardana and Perrot, that $Z \approx Z^{*}+2\left(a_{i i} / \lambda_{S}\right)^{2}\left[e^{\left(E_{\mathrm{hop}}-\mu\right) / k_{B} T}+1\right]^{-1}$ [28], where $\lambda_{S}$ is the short-range screening length [24] and $\mu$ is the electronic chemical potential. $E_{\mathrm{hop}} \approx-Z^{*} e^{2} / 4 \pi \epsilon_{0} \lambda_{S}$ is the so-called mobility edge: Localized electrons with energy $E>E_{\text {hop }}$ are bound to ion clusters. All of these electrons do not strictly contribute to the overall conductivity or opacity of the medium, but they participate in both short- and long-range screening of the ions. Indeed, assuming $\lambda_{S} \sim a_{i i}$ and $Z^{*}=0.1$ for $t=t_{0}+8 \mathrm{~ns}$, we get $Z \sim$ 1.65 , which is in good agreement with the fit to the experimental data. A good match $(Z \sim 0.6)$ for $t=t_{0}+2 \mathrm{~ns}$, closer to the insulator state, requires a longer screening length $\lambda_{S} \sim 1.8 a_{i i}$. While still a working hypothesis, the correlation length $L_{\text {corr }}$ may be thus representative of this electron hopping among neighboring ions.

Since carbon and hydrogen ions have a large difference in the atomic number, we need to verify that on average the ion-ion coupling can be represented in terms of a singlecomponent ionic fluid. Indeed, Kritcher et al. [12] have assumed that, in $\mathrm{LiH}$ mixtures, the correlations are essentially determined by the $\mathrm{Li}$ ions with the hydrogen playing no significant role. As discussed in Ref. [29] for the case of a mixture, we can define the equivalent to Eq. (1) as $\bar{S}_{\text {elas }}(k)=\left|\bar{f}_{I}(k)+\rho(k)\right|^{2} \bar{S}_{i i}(k)$, where $\bar{f}_{I}(k)$ and $\bar{S}_{i i}(k)$ corresponds to properly averaged structure factors [29]. The screening density $\rho(k)$ is taken to be the same for each ionic species, and it is calculated within the linear response formalism, which has been proven to successfully describe small wave number correlations in compressed lithium samples [23]. In order to assess the validity of an average one-component approximation, we have conducted single- and multicomponent hypernetted chain (HNC) simulations [30]. We have used two versions of the HNC modeling of the plasma: The first one treats the ions (with either an average charge state or two ion species) with a linearly screened (Yukawa) potential, which has been shown to successfully reproduce previous scattering data in WDM lithium [23]. In the second version of the HNC simulation (see Fig. 4), we have instead treated the electrons as a rigid neutralizing background (the OCP model). In both cases, we see that differences between the single- and the multispecies calculations are modest in the wavelength range of interest, indicating that an average representation of the correlation length is indeed appropriate for these plasma conditions.

In summary, in this Letter we have demonstrated that by time-resolved picosecond x-ray scattering we can obtain snapshots of the correlation length in WDM. In particular, in dense, shock-driven plastic samples, strong coupling results in the formation of short-range order within three atomic sites. This results are important for improving our understanding of the thermodynamics of complex correlated systems and for conditions relevant to both laboratory astrophysics and inertial confinement fusion research.

We thank the essential support from the LULI technical team during the experimental campaign. Fruitful discussions with C. Blancard and G. Faussurier (CEA) during the preparation of this manuscript are acknowledged. This work was funded by the EU program Laserlab-Europe (lulinano2000001314). Partial support from the Engineering and Physical Sciences Research Council (Grant No. EP/G007187/1) and the Science and Technology Facilities Council of the United Kingdom is also acknowledged.

[1] J. Lindl et al., Phys. Plasmas 11, 339 (2004).

[2] G. Huser et al., Phys. Plasmas 12, 060701 (2005).

[3] D. Saumon et al., Astrophys. J. 99, 713 (1995).

[4] T. Guillot, Science 286, 72 (1999).

[5] G. Gregori et al., Phys. Rev. E 67, 026412 (2003).

[6] S. H. Glenzer et al., Phys. Rev. Lett. 90, 175002 (2003).

[7] A. Ravasio et al., Phys. Rev. Lett. 99, 135006 (2007).

[8] S. H. Glenzer et al., Phys. Rev. Lett. 98, 065002 (2007).

[9] D. Riley et al., Phys. Rev. Lett. 84, 1704 (2000).

[10] E. Garcia-Saiz et al., Phys. Rev. Lett. 101, 075003 (2008).

[11] G. Gregori et al., Phys. Rev. Lett. 101, 045003 (2008).

[12] A. L. Kritcher et al., Science 322, 69 (2008).

[13] J. Chihara, J. Phys. F 17, 295 (1987).

[14] J. Chihara, J. Phys. Condens. Matter 12, 231 (2000).

[15] A. Höll et al., Eur. Phys. J. D 29, 159 (2004).

[16] D. A. Pepler et al., in Central Laser Facility Annual Report, 2006 (unpublished).

[17] H.-S. Park et al., Phys. Plasmas 13, 056309 (2006).

[18] E. Garcia-Saiz et al., Rev. Sci. Instrum. 78, 095101 (2007).

[19] P. Celliers et al., Appl. Phys. Lett. 73, 1320 (1998).

[20] R. Ramis and J. Meyer-Ter-Vehn, Comput. Phys. Commun. 49, 475 (1988).

[21] S. Lyon and J. Johnson, Los Alamos National Laboratory Report No. LA-UR-92-3407, 1992.

[22] G. Gregori et al., High Energy Density Phys. 3, 99 (2007).

[23] E. Garcia-Saiz et al., Nature Phys. 4, 940 (2008).

[24] S. Ichimaru, Rev. Mod. Phys. 54, 1017 (1982).

[25] G. D. Tsakiris and K. Eidmann, J. Quant. Spectrosc. Radiat. Transfer 38, 353 (1987).

[26] R. M. More et al., Phys. Fluids 31, 3059 (1988).

[27] H. Sawada et al., Phys. Plasmas 14, 122703 (2007).

[28] M. W. C. Dharma-Wardana and F. Perrot, Phys. Rev. A 45, 5883 (1992).

[29] G. Gregori et al., J. Quant. Spectrosc. Radiat. Transfer 99, 225 (2006).

[30] K. Wünsch et al., Phys. Rev. E 77, 056404 (2008). 